\title{
Effects of APETALA2 on embryo, endosperm, and seed coat development determine seed size in Arabidopsis
}

\author{
Masa-aki Ohto • Sandra K. Floyd • Robert L. Fischer • \\ Robert B. Goldberg $•$ John J. Harada
}

Received: 8 June 2009/Accepted: 1 October 2009/Published online: 25 October 2009

(c) The Author(s) 2009. This article is published with open access at Springerlink.com

\begin{abstract}
Arabidopsis APETALA2 (AP2) controls seed mass maternally, with ap 2 mutants producing larger seeds than wild type. Here, we show that AP2 influences development of the three major seed compartments: embryo, endosperm, and seed coat. AP2 appears to have a significant effect on endosperm development. ap2 mutant seeds undergo an extended period of rapid endosperm growth early in development relative to wild type. This early expanded growth period in ap 2 seeds is associated with delayed endosperm cellularization and overgrowth of the endosperm central vacuole. The subsequent period of moderate endosperm growth is also extended in ap 2 seeds largely due to persistent cell divisions at the endosperm
\end{abstract}

Communicated by Scott Russell.

M. Ohto · S. K. Floyd · J. J. Harada ( $ه)$

Department of Plant Biology, College of Biological Sciences,

University of California, One Shields Avenue,

Davis, CA 95616, USA

e-mail: jjharada@ucdavis.edu

R. L. Fischer

Department of Plant and Microbial Biology,

University of California, Berkeley, CA 94720, USA

R. B. Goldberg

Department of Molecular, Cell, and Developmental Biology,

University of California, Los Angeles, CA 90024, USA

Present Address:

M. Ohto

Mendel Biotechnology, Inc., 3935 Point Eden Way,

Hayward, CA 94545-3720, USA

Present Address:

S. K. Floyd

School of Biological Sciences, Monash University,

Clayton Campus, Melbourne, VIC 3800, Australia periphery. The effect of AP2 on endosperm development is mediated by different mechanisms than parent-of-origin effects on seed size observed in interploidy crosses. Seed coat development is affected; integument cells of ap2 mutants are more elongated than wild type. We conclude that endosperm overgrowth and/or integument cell elongation create a larger postfertilization embryo sac into which the ap 2 embryo can grow. Morphological development of the embryo is initially delayed in ap 2 compared with wild-type seeds, but ap 2 embryos become larger than wild type after the bent-cotyledon stage of development. ap2 embryos are able to fill the enlarged postfertilization embryo sac, because they undergo extended periods of cell proliferation and seed filling. We discuss potential mechanisms by which maternally acting AP2 influences development of the zygotic embryo and endosperm to repress seed size.

Keywords AP2 - Maternal control - Seed development . Seed mass

\section{Introduction}

The seed consists of three major compartments, the embryo, endosperm, and seed coat, that originate from different cells of the ovule and possess different complements of maternal and paternal genomes (Bewley and Black 1994; Ohto et al. 2008). Seed development proceeds through two distinct phases during which growth of the three compartments is coordinated. During the early morphogenesis phase, the embryo undergoes a series of differentiation events in which the plant body plan is established with formation of the embryonic tissue and organ systems. It is also during this phase that the 
endosperm mother cell initially undergoes nuclear divisions without cytokinesis to form a syncytium (Brown et al. 1999). Syncytial nuclei are sequestered into individual endosperm cells through the process of cellularization, and the endosperm continues to grow through periclinal cell divisions at the periphery of the endosperm. Later in embryogenesis during the maturation phase, the embryo and endosperm accumulate reserves such as storage lipids and proteins, and the embryo acquires the ability to withstand desiccation (Harada 1997; Vicente-Carbajosa and Carbonaro 2005). In plants such as Arabidopsis thaliana (L.) Heynh, the endosperm is largely consumed by the developing embryo such that only a few endosperm cell layers remain in the mature seed.

The final size or mass attained by a seed is influenced by a variety of cellular processes. One pathway involves parent-of-origin effects on seed size. Interploidy crosses between diploid and polyploid plants produce offspring with an excess of either maternal or paternal genomes, which cause an under- or over-proliferation of endosperm nuclei, an acceleration or delay in the onset of endosperm cellularization, and the production of smaller or larger seeds, respectively (Haig and Westoby 1991; Scott et al. 1998). Reciprocal crosses of wild-type plants with hypomethylated plants defective in the expression of MET1, encoding a DNA methyltransferase, phenocopies interploidy crosses (Adams et al. 2000), suggesting that parent-of-origin effects involve DNA methylation. A second pathway involves genes, including IKU and MINI3, expressed in the endosperm that act to control endosperm growth and seed size. A third potential pathway involves genes that act in the maternal sporophyte to influence seed size, including $A P 2, T T G 2$, and $A R F 2$. Loss-of-function mutations in TTG2 and ARF2 affect the lengths and number of integument cells of the ovule, respectively (Garcia et al. 2005; Schruff et al. 2006), and the influence of these genes on seed size has been primarily attributed to their effect on the size of the postfertilization embryo sac.

AP2 (APETALA2), the defining member of the AP2 DNA binding domain class of transcription factors (Jofuku et al. 1994), is involved in controlling seed size (Jofuku et al. 2005; Ohto et al. 2005). AP2 was identified originally as a gene required to specify floral organ and floral meristem identity and to control ovule and seed coat development (Kunst et al. 1989; Irish and Sussex 1990; Bowman et al. 1991, 1993; Schultz and Haughn 1993; Okamuro et al. 1993). Although its most conspicuous functions are in flower development, AP2 RNA is detected in seedlings, leaves and stems in addition to flowers, suggesting its function in other processes (Jofuku et al. 1994). Loss-offunction mutations in ap 2 affect seed mass maternally, although it has been suggested that $A P 2$ acts in the endosperm to influence seed size (Jofuku et al. 2005; Ohto et al.
2005). The ap 2 mutation causes an increase in both the number and size of cells in the mature embryo and in the accumulation of storage proteins and lipids in mutant seeds when compared with wild type. We showed previously that AP2 also affects sugar composition in seeds and hypothesized that differences in the hexose to sucrose ratio in wildtype and ap 2 mutant seeds might underlie, in part, the large seed phenotype (Ohto et al. 2005).

To obtain clues about the mechanisms by which AP2 controls seed size, we analyzed seed development in the ap 2 mutant. We expanded on previous studies that analyzed the effects of the $a p 2$ mutation on mature, dry seeds (Jofuku et al. 2005; Ohto et al. 2005) and showed that AP2 has a major effect on the early phase of endosperm development and on the size of the postfertilization embryo sac into which the embryo will grow. We show further that AP2 affects the timing of embryo development and influences cell proliferation and the timing of the maturation phase in the seed. The influence of these processes on seed size is discussed.

\section{Materials and methods}

\section{Plant materials}

A strong ap2 mutant allele, ap2-7 (Kunst et al. 1989), was used for all experiments. ap2-7 and transgenic plants carrying $\beta C G$ :GUS (Hirai et al. 1994) and CycB1;1:GUS (Colon-Carmona et al. 1999) are of the Columbia ecotype, whereas the transgenic plant with $O L E$ :GUS is of the C24 ecotype (Kwong 2003). The met1-6 mutation in the Col-gl ecotype was introgressed into the Columbia ecotype with six backcrosses (Xiao et al. 2003). For all experiments, wild-type and ap 2 plants were grown together for the same period of time at $22^{\circ} \mathrm{C}$ under continuous light.

Seeds were staged by days after hand pollination of emasculated flowers according to procedures described previously (Ohto et al. 2005). Pollinations for transgene assays were done between male plants homozygous for $\beta C G: G U S, C y c B 1 ; 1: G U S$ or $O L E: G U S$ and non-transgenic wild-type or ap2 females, unless otherwise indicated.

\section{Microscopy}

Developing seeds dissected from siliques were dehydrated in ethanol and cleared with methyl salicylate (West et al. 1994). One DAP, ovules were considered to be fertilized if they contained embryos with two or more cells and/or endosperm with two or more nuclei. To observe endosperm nuclei using differential interference contrast microscopy, seeds at 6 DAP were fixed in ethanol:acetic acid (3:1) for $1 \mathrm{~h}$, rehydrated in an ethanol series, and cleared in Hoyer's 
solution. At 1-5 days, seeds were cleared in Hoyer's solution (Ohto et al. 2005). To count endosperm nuclei, optical sections of the entire endosperm at $7-10-\mu \mathrm{m}$ intervals were photographed. The positions of endosperm nuclei were marked on a plastic sheet to monitor their positions.

For plastic sections, siliques were cut transversely into two pieces and fixed in triple fixative (100 mM PIPES, $10 \mathrm{mM}$ EGTA, $2 \mathrm{mM} \mathrm{MgSO}_{4}, \mathrm{pH}$ 6.8, with $1 \%$ paraformaldehyde, $1.5 \%$ glutaraldehyde and $4 \%$ acrolein). Fixed tissues were rinsed with PIPES buffer and subsequently dehydrated through an ethanol series to $75 \%$ ethanol and stored at $4^{\circ} \mathrm{C}$ until needed. Following dehydration to $95 \%$ ethanol, tissues were embedded in plastic, sectioned at 5- $\mu \mathrm{m}$ thickness and stained with toluidine blue as described previously (Floyd et al. 1999). Serial sections through three to four siliques were analyzed for each seed stage and genotype.

The areas within cleared or sectioned seeds were measured using NIH IMAGE analysis software (http://rsbweb. nih.gov/nih-image/). Seed sizes from crosses with met1 mutant males are the sum of the width and length of developing 13 DAP seeds.

GUS activity was analyzed histochemically as described previously (Dietrich et al. 1989). Tissues were incubated in the dark for $12 \mathrm{~h}$ at $37^{\circ} \mathrm{C}$. Embryos and endosperm/seed coat were separated before staining. Stained material was fixed, rehydrated, and cleared in Hoyer's solution. GUS staining intensities shown in Figs. 5 and 6 were determined empirically.

In vitro culture of embryo and endosperm with sugars

Embryos from 15 to 20 seeds from the same silique were cultured in basal media (Millerd et al. 1995) containing either high hexose $(75 \mathrm{mM}$ glucose, $75 \mathrm{mM}$ fructose and $15 \mathrm{mM}$ sucrose) or high sucrose (110 mM sucrose). Embryos were cultured under continuous light at $22^{\circ} \mathrm{C}$ for 3 days.

\section{Results}

Seed size comparison during development of ap2 and wild-type seeds

We compared developmental changes in the morphology and size of ap2 mutant and wild-type seeds to identify factors that cause ap2 seeds to become larger than wild type. Figure 1a-m shows longitudinal sections through cleared seeds, and Fig. 1n summarizes quantitative changes in wild-type and ap 2 seed sizes. ap 2 and wild-type seeds first display a significant size difference at 7 DAP. ap2 and wild-type seeds both underwent an early rapid phase of seed growth that was followed by a moderate growth phase. However, the rapid growth period was extended in ap2 mutants relative to wild type (Fig. 1n), resulting in the larger size of ap 2 seeds. The ap 2 mutation also caused a significant change in seed shape. At 7 DAP, the length to width ratios of ap2 and wild-type seeds were $1.03 \pm 0.15$ $(n=30)$ and $1.71 \pm 0.15(n=40, P<0.001)$, respectively, indicating that ap2 seeds are more round in shape than wild type. Thus, the altered shape and larger mass of ap2 seeds is determined, at least in part, early in seed development.

\section{Effects of AP2 on embryo development}

An unexpected feature of ap2 seed development was that morphological development of embryos was initially slower than wild type (Fig. 1a-c, f-h). Analysis of cleared seeds showed that the percentage of fertilized ap2 and wild-type seeds was similar at 1 DAP $(64.5 \%, n=161$, and $60.5 \%, n=124$, respectively; see "Materials and methods"), suggesting female gametophytes of both genotypes were fertilized with similar efficiencies. Figure 2 shows that morphological development of ap2 embryos was delayed soon after fertilization. At 2 DAP, ap2 embryos were at the one to four-celled embryoproper stage, whereas wild-type embryos were at the two to eight-celled embryo-proper stage (Fig. 2a). The delay in embryo morphogenesis was also observed at 3, 4 and 5 DAP (Fig. 2b-d), although no obvious difference in the cellular arrangement of ap2 and wild-type embryos was apparent (Fig. 1 and data are not shown). The slower rate of ap2 embryo development was confirmed in several independent experiments. Reciprocal crosses showed the rate of embryo development was determined by the female rather than male genotype (Fig. 2a-d). For example, embryos derived from a cross between homozygous ap2 mutant females and wild-type males developed at a similar rate as do the embryos from self-crossed ap2 homozygous mutant plants. These results indicate that $A P 2$ affects embryo development maternally and that the delay in morphological development of ap2 embryos occurs soon after fertilization at the earliest stages of embryo development.

Because morphological development of ap2 embryos was delayed, mutant embryos were smaller than wild type at the same DAP until approximately 8-9 DAP when both were at the bent-cotyledon stage (Fig. 1d, i). By 11-12 DAP, wild-type embryos were essentially at their final size (Fig. 1k), whereas ap2 embryos continued to grow in size until approximately 14-15 DAP (Fig. 1m). ap2 and wild-type embryos completely filled the postfertilization embryo sac at 19.9 $\pm 0.9 \mathrm{DAP}$ and 17.5 $\pm 0.5 \mathrm{DAP}$, respectively. Thus, ap 2 embryos develop similar to wild 

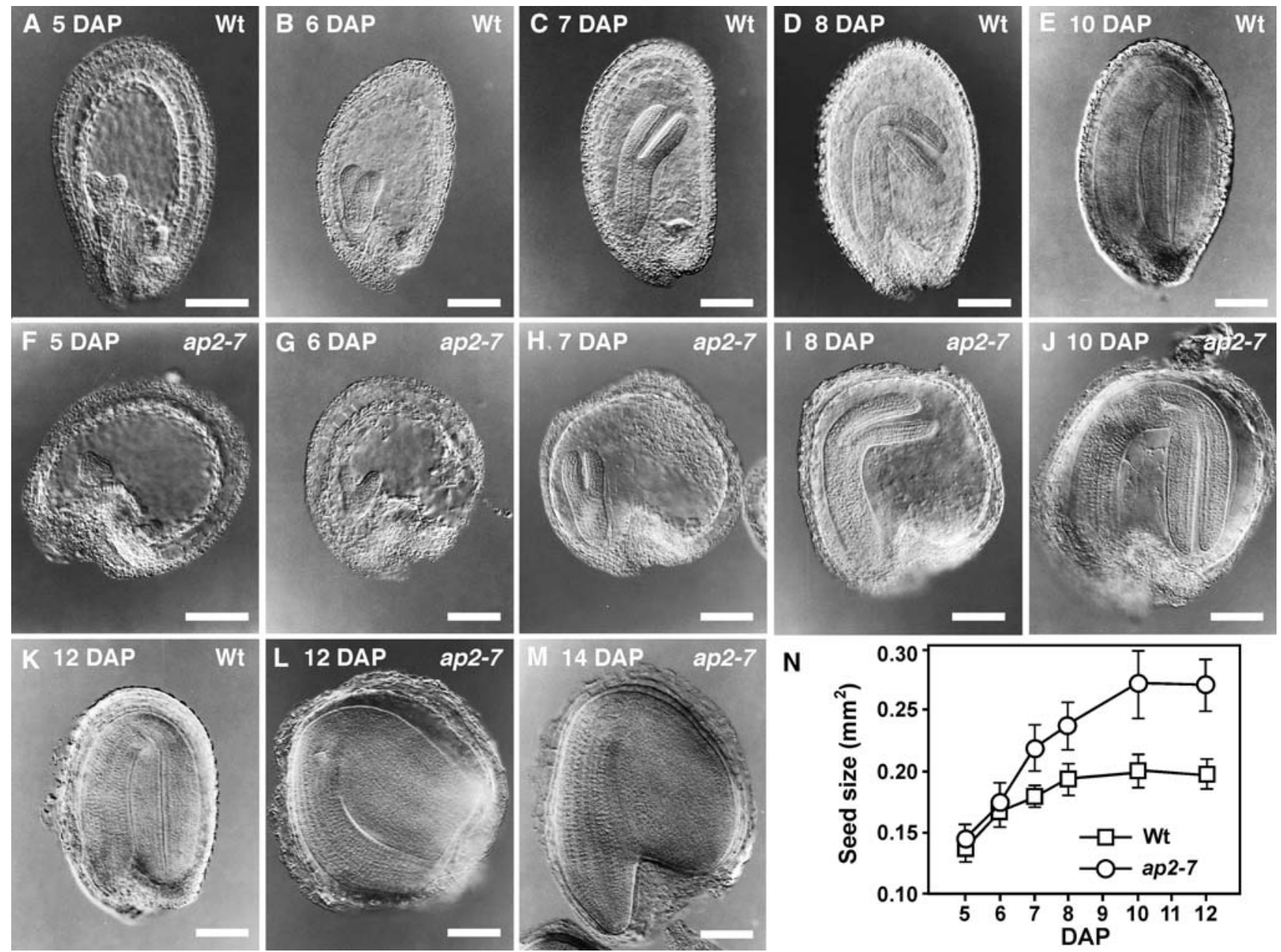

Fig. 1 ap2 mutation causes changes in embryo and seed development. Optical sections through developing wild-type $(\mathbf{a}-\mathbf{e}, \mathbf{k})$ and ap2 seeds $(\mathbf{f}-\mathbf{j}, \mathbf{l}, \mathbf{m})$ at the indicated DAP. Seeds from three to five siliques at each time point were harvested, fixed and cleared and representative optical sections are shown. $\mathbf{n}$ Average maximal size (area) through developing seeds calculated using imaging software. Each value represents the mean obtained from measurement of 30-45 seeds and standard deviations. Bars $=100 \mu \mathrm{m}$ type during the morphogenesis phase except for the slow rate of cell proliferation before $8 \mathrm{DAP}$, and they continue to grow for a longer period of time than wild type after 8 DAP.

\section{AP2 affects early endosperm development}

The precocious increase in ap2 seed size and delayed development of ap2 embryos relative to wild type early in seed development suggested a role for the endosperm in determining ap2 seed size. We analyzed histological sections through ap2 and wild-type seeds and identified similarities between the overall patterns of ap2 and wild-type endosperm development, as shown in Fig. 3. For example, endosperm of both genotypes possessed a large central vacuole early in seed development (Fig. 3a, b and e, f), developed initially as a syncytium (Fig. 3a, b and e, f), cellularized as a wave from the micropylar to the chalazal pole (Fig. 3b, c, i and g, h, j-l), retained a small region that remained uncellularized (Fig. $3 \mathrm{i}$ and 1) and underwent periclinal cell divisions that began in the micropylar pole and expanded into the peripheral region (Fig. 3n-p).

A major feature distinguishing ap2 and wild-type endosperm development was the size and persistence of the central vacuole. In comparing ap2 and wild-type seeds at the same DAP (Fig. 3c and g, d and h, i and j) or at the same embryo stage (Fig. $3 \mathrm{c}$ and $\mathrm{h}$ ), the size of the ap2 endosperm central vacuole was larger than that of wild type. Figure 4 shows the cross-sectional area of the ap2 and wild-type endosperm central vacuole was maximal at 6 and 5 DAP, respectively, and the ap 2 central vacuole was approximately twice the area of wild type. Figures $3 \mathrm{~d}, \mathrm{i}$ and $\mathrm{k}, 1$ and 4 show that the ap 2 endosperm central vacuole persisted for a longer period of time than wild type. Thus, central vacuole size correlates with endosperm size in the two genotypes. 


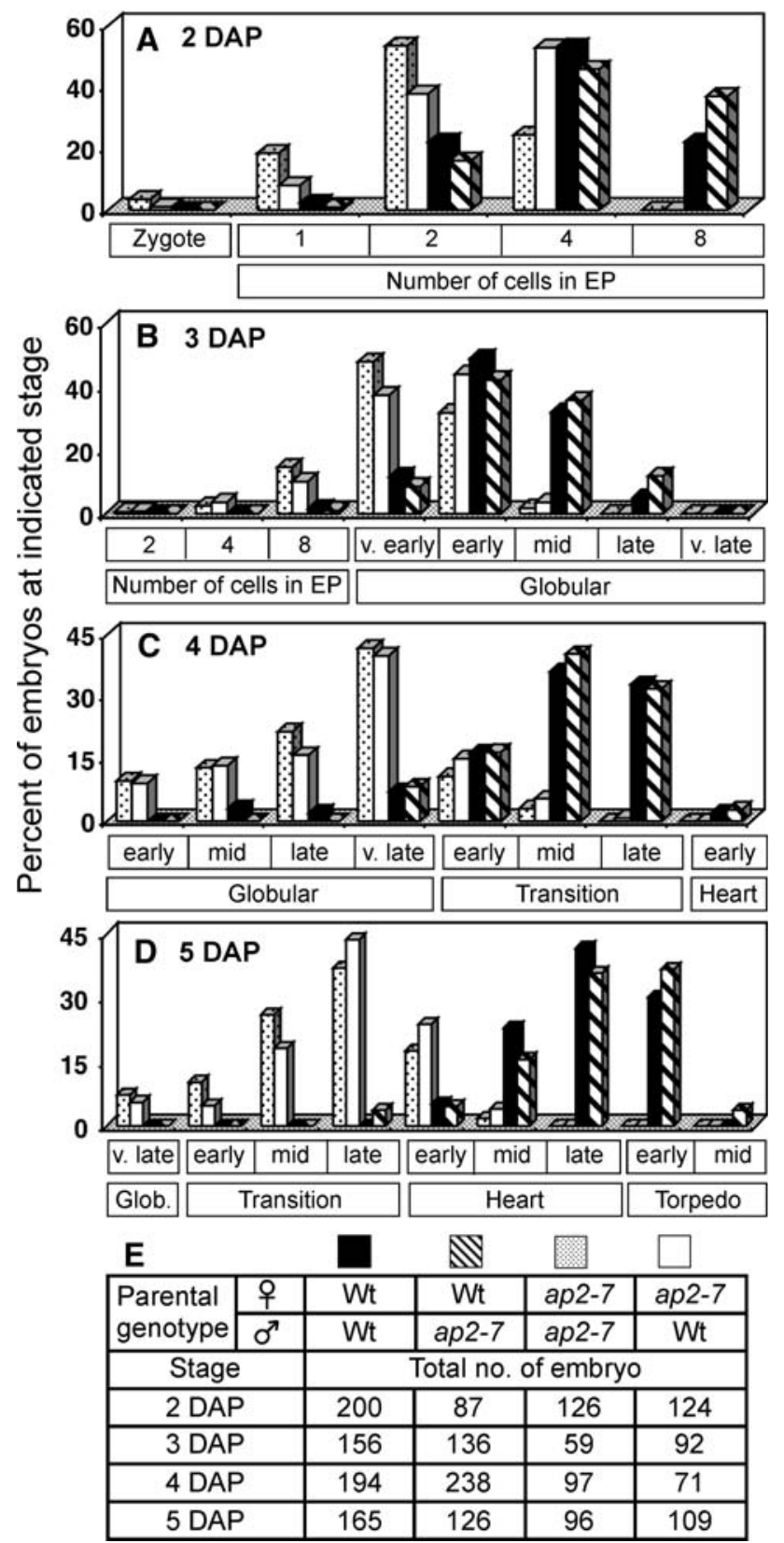

Fig. 2 Morphological development of embryos is delayed in ap2 mutants. a-d Percent of embryos at a particular morphological stage at the indicated DAP. Developing seeds were harvested at 2-5 DAP, cleared and staged. For each cross, three to six siliques were harvested at each time point. After the eight-celled, embryo-proper stage, embryo morphology was classified into five subclasses of globular stage, three subclasses each of transition and heart stages, and two of torpedo stage. e Number of seeds analyzed in (a-d). EP embryo proper

A second difference was that cellularization of the ap2 endosperm syncytium began and ended later in development than wild type. ap 2 endosperm cellularization initiated when embryos were at the middle-heart stage at approximately 6 DAP (data not shown, Fig. $3 \mathrm{~g}$ shows cellularization in late-heart stage seeds), whereas wild-type endosperm cellularization began when embryos were at the early-heart stage (data not shown, Fig. 3b shows cellularization at the mid-heart stage) at 5 DAP. Endosperm cellularization was essentially complete in ap2 and wild-type seeds containing bent-cotyledon stage embryos at 9 and 7 DAP, respectively (Fig. $3 \mathrm{~m}$ and d; Table 1). Together, these results indicate endosperm size, particularly vacuole volume, and the delay in endosperm cellularization correlate closely with $a p 2$ seed size relative to wild type.

A final difference between ap2 and wild-type endosperm is their cell size. The average cross-sectional area of ap2 and wild-type endosperm cells at 7 DAP was $1,030 \pm 145 \mu \mathrm{m}^{2}(n=832,13$ seeds $)$ and $740 \pm 66 \mu \mathrm{m}^{2}$ ( $n=355,5$ seeds), respectively. The ratio of ap2 to wildtype endosperm cell area was similar to the corresponding ratio of embryo cell area determined previously (1.4 and 1.3, respectively; Ohto et al. 2005). Together, these results suggest that changes in endosperm development account, in part, for the effect of the ap 2 mutation on seed size.

We investigated how the seed coat contributes to changes in ap 2 seed size and showed that the average length of seed coat epidermal cells in ap2 and wild-type seeds at 8 DAP were $41 \pm 4.3$ and $30 \pm 3.6 \mu \mathrm{m}$, respectively. This result suggests that $a p 2$ integument cells were elongated relative to wild type.

ap2 mutation causes a delay in the onset of seed maturation in the embryo and endosperm

We monitored the activities of GUS reporter genes fused with the promoters of genes encoding the $\alpha^{\prime}$ subunit of soybean $\beta$-conglycinin ( $\beta C G$ :GUS; Hirai et al. 1994) and the lipid-body protein, oleosin (OLE:GUS; Kwong 2003) to determine whether AP2 affects the timing of the onset of the maturation phase. The promoters are active predominately during the maturation phase of seed development in Arabidopsis, and their activities are markers for this phase.

Dissected embryos and the remaining endosperm/seed coat fractions were stained separately for GUS activity. GUS staining was first detectable in wild-type (Fig. 5a, e) and ap2 (Fig. 5c, h) embryos containing the $\beta C G$ :GUS gene at 8 and 9 DAP, respectively, and was intense in embryos at 9 and 10 DAP, respectively (Fig. 5b, g and d). The 1-day difference between the start of GUS staining in wild-type and ap2 embryos was observed in replicate experiments, although staining began at 9 and 10 DAP, respectively, in some experiments. Similar results were obtained with embryos containing the $O L E$ : GUS transgene. As shown in Fig. 5i-k, the oleosin promoter became active in ap2 embryos approximately 1 day later than wild type. We also showed that the ap2 mutation affects the onset of the maturation phase in endosperm. The endosperm/seed coat fraction from $\beta C G$ :GUS and $O L E$ :GUS seeds stained 

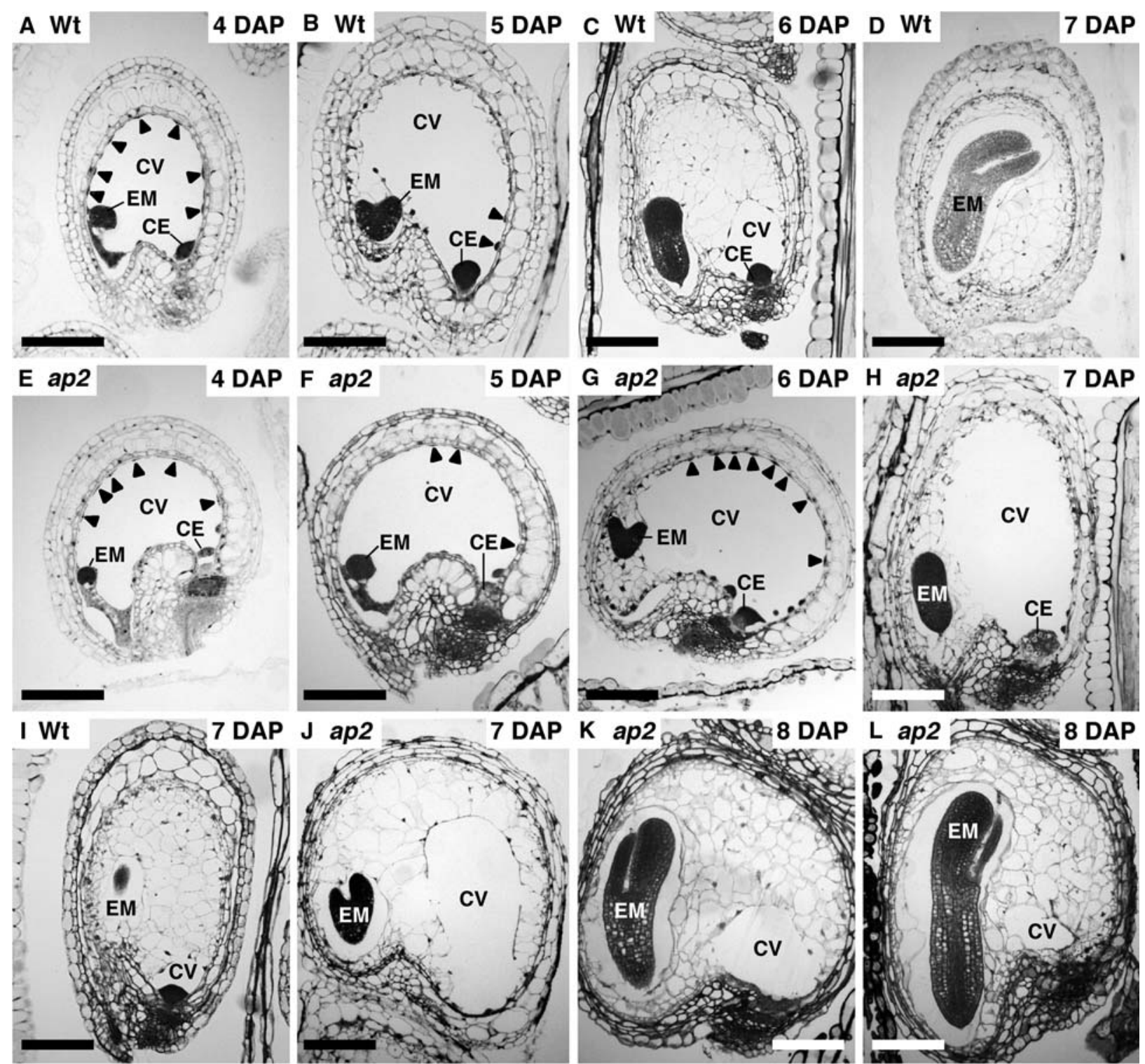

7 DAP
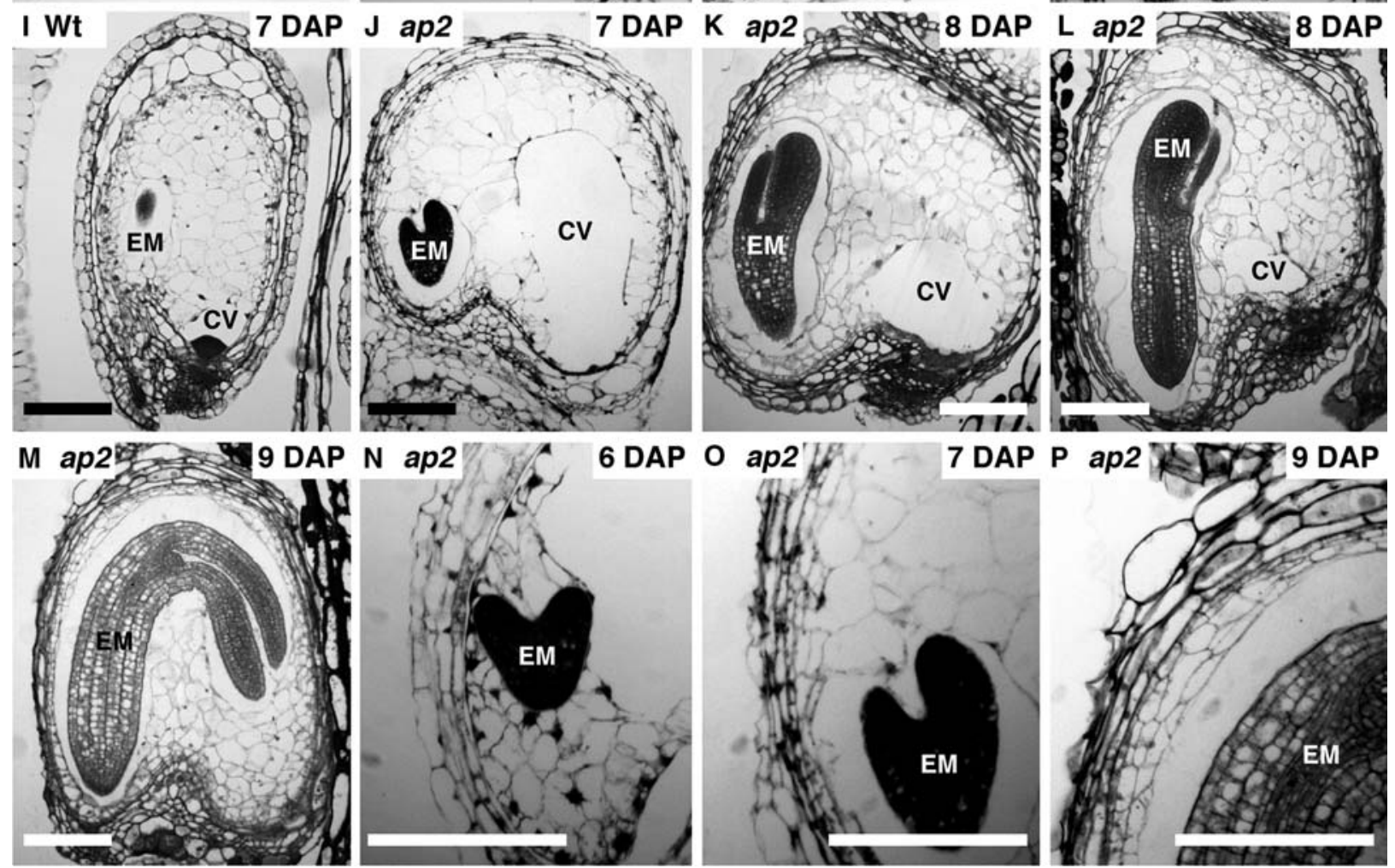

Fig. 3 ap2 mutation affects the timing of endosperm cellularization and endosperm central vacuole size. Longitudinal sections through wild type $(\mathbf{a}-\mathbf{d}, \mathbf{i})$ and ap 2 mutants $(\mathbf{e}-\mathbf{h}, \mathbf{j}-\mathbf{p})$ seeds at the indicated DAP. Seeds $(\mathbf{a}-\mathbf{m})$ are oriented with the micropyle on the left. Embryo stages are as follows: a early transition; b mid-heart; $\mathbf{c}$ earlylinear cotyledon; d early-bent cotyledon; e early-globular; f mid

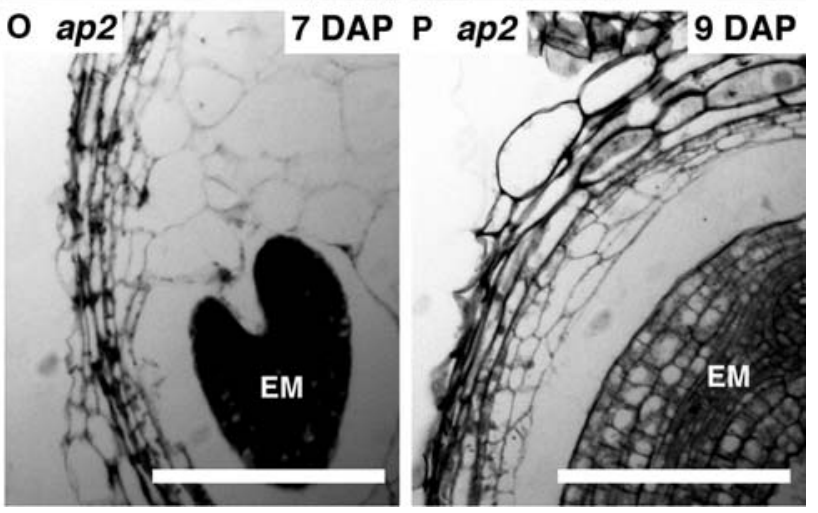

transition; $\mathbf{g}$ late heart; $\mathbf{h}$ early-linear cotyledon; $\mathbf{i}$ mid-linear cotyledon; $\mathbf{j}$ early or mid-linear cotyledon; $\mathbf{k}, \mathbf{l}$ late-linear cotyledon; $\mathbf{m}$ late-bent cotyledon. $\mathbf{n}-\mathbf{p}$ Enlargement of part of $(\mathbf{g}),(\mathbf{j})$ and $(\mathbf{m})$, respectively. Arrowheads show endosperm nuclei. $C E$ chalazal endosperm, $C V$ central vacuole, $E M$ embryo. $B a r=100 \mu \mathrm{m}$ 
approximately 1 day later in ap2 versus wild-type genotypes (Table 2). These results suggest the ap2 mutation causes an approximately 1-day delay in initiation of the maturation phase in both embryos and endosperm.

Effect of the ap2 mutation on cell proliferation

We determined when cell division became suppressed in ap2 relative to wild-type seeds as an independent marker for onset of the maturation phase. Cell proliferation is largely inhibited in the embryo and endosperm during this period (Harada 1997; Raz et al. 2001; Olsen 2004). We used a $C y c B 1 ; 1: G U S$ gene that is active specifically during the $\mathrm{G} 2$ to M transition of the cell cycle (Colon-Carmona et al. 1999). As shown in Fig. 6a, c, and summarized in Fig. 6e, g, i, wildtype embryos stained weakly for GUS activity at 9 DAP, and this staining level decreased until 10 DAP when GUS activity was nearly undetectable. By contrast, Fig. 6b, d, f, h, $\mathrm{j}$ show that ap2 embryos stained for GUS activity more intensely than wild-type embryos of the same size and at the same DAP. These results suggest that cell proliferation continues later in development in ap2 versus wild-type embryos. Consistent with this interpretation, the cross-sectional areas of cleared, ground tissue cells from the axes of ap2 and wild-type embryos at 9 DAP were $216 \pm 12 \mu \mathrm{m}^{2}$ $(n=179,9$ embryos $)$ and $238 \pm 15 \mu^{2}(n=167,9$ embryos), respectively. Because embryos of both genotypes were of similar size at 9 DAP (see Figs. 5, 6) and mature ap2 embryos have $40 \%$ more cells than wild type (Ohto et al. 2005), this result suggests that cell division occurs more extensively in ap 2 than wild-type embryos after 9 DAP.

Cell division in the endosperm also persisted later in development in ap2 versus wild-type seeds. Although most endosperm cells form as a result of cellularization of the syncytium, periclinal cell divisions occur at the periphery of the cellularized endosperm, forming successive layers of endosperm cells (Fig. 3o, p; Brown et al. 1999). Figure 7a-d, shows that $C y c B 1 ; 1$ promoter activity was suppressed earlier during development of wild-type versus ap2 endosperm. Thus, consistent with the activities of seed protein promoters, these results suggest the onset of the maturation phase is delayed in ap2 seeds.

We showed previously that the ratio of hexose (D-glucose and D-fructose) to sucrose, normally high during the morphogenesis phase, remains elevated later in ap2 seed development than in wild type (Ohto et al. 2005). Because a high hexose to sucrose ratio promotes cell division (Weber et al. 1996), we postulated AP2 controls cell proliferation in developing seeds, in part, through its effect on sugar metabolism (Ohto et al. 2005). To examine the effect of sugars on embryo cell proliferation, we cultured 8 DAP wild-type embryos containing the $C y c B 1 ; 1: G U S$ gene in medium with different ratios of hexose to sucrose.

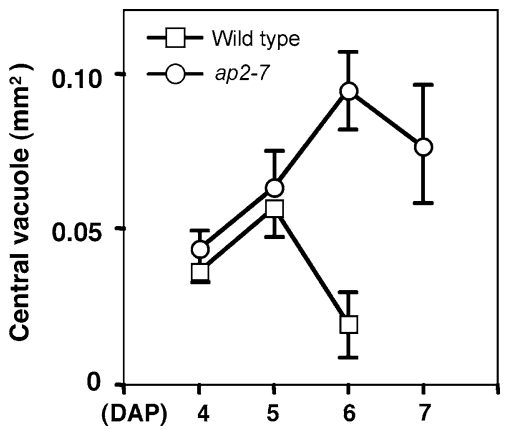

Fig. 4 Central vacuole of ap2 endosperm is larger than wild type. Maximal central vacuole cross-sectional area was measured from serial sections through seeds similar to those shown in Fig. 3. Mean values and standard deviations are plotted

Table 1 ap2 endosperm cellularizes later than wild type

\begin{tabular}{|c|c|c|c|c|c|c|c|}
\hline \multirow[t]{2}{*}{ Genotype } & \multirow[t]{2}{*}{ DAP } & \multirow[t]{2}{*}{ None } & \multicolumn{5}{|c|}{ Extent of endosperm cellularization ${ }^{a}$} \\
\hline & & & Early & Middle & Late & Complete & $n$ \\
\hline WT & 4 & $100(a)^{b}$ & 0 & 0 & 0 & 0 & \\
\hline ap2 & 4 & 100 (e) & 0 & 0 & 0 & 0 & \\
\hline WT & 5 & 0 & $100(b)$ & 0 & 0 & 0 & \\
\hline ap2 & 5 & 100 (f) & 0 & 0 & 0 & 0 & \\
\hline WT & 6 & 0 & 3 & 10 & $54(\mathrm{c})$ & 33 (i) & \\
\hline ap2 & 6 & 13 & $87(\mathrm{~g})$ & 0 & 0 & 0 & \\
\hline WT & 7 & 0 & 0 & 0 & 0 & $100(\mathrm{~d})$ & \\
\hline ap2 & 7 & 1 & 43 (h) & $50(\mathrm{j})$ & 6 & 0 & \\
\hline
\end{tabular}

${ }^{a}$ Values indicate the percentage of seeds at the indicated stage of endosperm cellularization

${ }^{\mathrm{b}}$ Corresponds to the indicated panel in Fig. 3

Embryos cultured in medium with a high hexose to sucrose ratio (Fig. 8a) exhibited substantially more $C y c B 1 ; 1$ promoter activity than those cultured in high sucrose (Fig. 8b), suggesting cell proliferation in Arabidopsis embryos can be affected by sugar composition.

\section{$A P 2$ and parent-of-origin effects on seed size}

We analyzed the effects of the ap2 mutation on seed development to determine if there were similarities with parent-of-origin effects on seed size resulting from interploidy crosses or crosses involving mutants defective in the MET1 gene (Scott et al. 1998; Vinkenoog et al. 2000; Luo et al. 2000; Adams et al. 2000). Because endosperm nuclei proliferation correlates with seed size in interploidy crosses, we counted endosperm nuclei. Table 3 shows ap2 endosperm nuclei number was lower than wild-type at 3 DAP and did not differ significantly from wild type at 6 DAP. Thus, by contrast to seeds undergoing parent-oforigin effects on seed size that display endosperm 
Fig. 5 Activation of maturation phase-specific promoters is delayed in ap2 mutants. a-d Wild-type (a, b) and ap $2(\mathbf{c}, \mathbf{d})$ embryos with the $\beta-C G: G U S$ transgene at the indicated DAP stained for GUS activity. $\mathbf{e}-\mathbf{h}$ Embryos from crosses between wild-type $(\mathbf{e}, \mathbf{g}, \mathbf{i}, \mathbf{j})$ or ap 2 mutant $(\mathbf{f}, \mathbf{h}, \mathbf{k})$ females and $\beta$-CG:GUS (e-h) or OLE:GUS (i-k) males were stained for GUS activity. Histograms show the number of embryos of a given size (indicated by cotyledon width) at a specific DAP. For each size class, the proportion of embryos exhibiting a given intensity of GUS staining is represented, with red indicating the strongest staining and white representing unstained embryos. Unlike wild type, most ap2 embryos containing the $\beta C G$ :GUS transgene were not strongly stained at 9 DAP, but stained intensely at 10 DAP. Similarly, ap2 embryos containing the OLE:GUS transgene were not strongly stained at 8 DAP, but stained intensely at 9 DAP. Bar $=100 \mu \mathrm{m}$

overgrowth (Scott et al. 1998), ap2 mutants did not exhibit overproliferation of endosperm nuclei.

We analyzed genetic interactions between $A P 2$ and $M E T 1$, because parent-of-origin effects on endosperm development are mediated, in part, through DNA methylation (Vinkenoog et al. 2000; Luo et al. 2000; Adams et al. 2000). Wild-type female plants crossed with male plants heterozygous for the metl mutation produced two size classes of seeds: one equivalent to wild type and the other that was smaller (Fig. 9a). This result was consistent with other reports that fertilization with the paternal metl mutation results in smaller seed size (Adams et al. 2000; Xiao et al. 2006). Figure $9 \mathrm{~d}$ shows that pollination of ap2 females with male met 1 heterozygotes resulted in a range of seed sizes, some equivalent to those produced in a cross between ap 2 mutant females and wild-type male plants (Fig. 9c) and others that were smaller. We interpret these results to indicate that pollination of ap2 mutant females with met 1 pollen resulted in seeds intermediate in size between large seeds resulting from a cross of ap 2 mutant females with wild-type pollen and the small seeds from the wild-type female by metl pollen cross, suggesting an additive rather than an epistatic interactions between $A P 2$ and MET1. These results suggest that AP2 does not affect seed size through parent-of-origin effects on DNA methylation.

\section{Discussion}

Endosperm size is affected by the AP2 mutation

ap2 mutant females produce larger seeds and mature embryos with more cells and larger cells than wild type (Jofuku et al. 2005; Ohto et al. 2005). We analyzed the effects of the ap 2 mutation on seed development to obtain insight into the mechanisms by which AP2 controls seed size. Our findings suggest AP2 normally suppresses endosperm growth, with ap 2 mutants having a larger
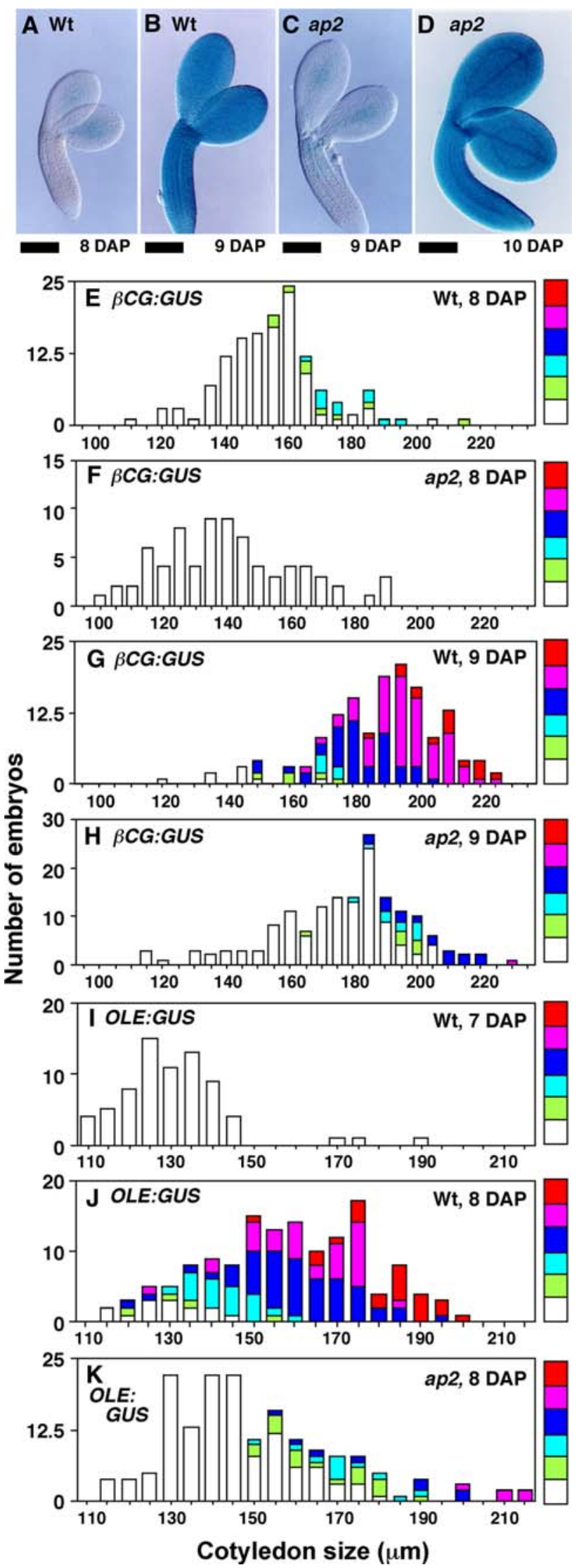
Table 2 Activation of maturation gene promoters is delayed in ap2 endosperm

\begin{tabular}{llllll}
\hline Transgene & Genotype & \multicolumn{3}{l}{$\begin{array}{l}\text { Percentage of seed with GUS-stained } \\
\text { endosperm }\end{array}$} \\
\cline { 3 - 6 } & & $8 \mathrm{DAP}$ & $9 \mathrm{DAP}$ & $10 \mathrm{DAP}$ & $11 \mathrm{DAP}$ \\
\hline$\beta C G: G U S$ & $\mathrm{WT}$ & $0(53)^{\mathrm{a}}$ & $59(90)$ & $95(87)$ & $100(92)$ \\
$\beta C G: G U S$ & ap2-7 & $0(61)$ & $0(91)$ & $67(91)$ & $76(83)$ \\
OLE:GUS & $\mathrm{WT}$ & $2(91)$ & $65(91)$ & $97(91)$ & $\mathrm{ND}$ \\
OLE:GUS & ap2-7 & $0(100)$ & $37(100)$ & $94(85)$ & $\mathrm{ND}$ \\
\hline
\end{tabular}

$N D$ Not determined

${ }^{a}$ Numbers of seeds analyzed

endosperm than wild type (Fig. 3). Specifically, AP2 appears to restrict the lengths of the rapid growth period early in seed development before endosperm cellularization (Figs. 1, 3, 4) and the moderate growth period resulting from periclinal cell divisions at the periphery of the endosperm later in seed development (Figs. 3, 7). Our results are consistent with reports showing that the size attained by the endosperm syncytium early in seed development appears to be a major determinant of seed size (Boisnard-Lorig et al. 2001) and that mutations that alter seed size cause changes in endosperm size primarily during the period of early endosperm growth (Garcia et al. 2005).

Two aspects of the mutant phenotype offer potential insights into the mechanisms by which AP2 suppresses endosperm expansion. First, the endosperm vacuole achieved a larger size and persisted later in development in ap2 mutant than in wild-type seeds (Figs. 3, 4). Retention of a large vacuole during endosperm cellularlization in ap 2 mutants appears to correlate with the increase in endosperm size observed early in seed development (Figs. 1, 3, 4). Because plant cell expansion is generally driven by turgor pressure determined by the water content of the vacuole, this result opens the possibility that AP2 functions to suppress endosperm vacuole size, thus restricting overall endosperm growth. Second, initiation of syncytial endosperm cellularization is delayed 1 day in ap2 mutants compared with wild type (Figs. 3, 4), suggesting AP2 acts indirectly to control the timing of endosperm cellularization. There is a strong correlation between precocious and delayed cellularization and endosperm undergrowth and overgrowth, respectively (Scott et al. 1998; Garcia et al. 2003; Kang et al. 2008), although no direct relationship has been demonstrated.

The conclusion that the effect of AP2 on endosperm development, in part, underlies its effect on seed size suggests maternally acting AP2 functions indirectly to control endosperm development. One potential explanation is that the effect of AP2 on sugar composition may affect endosperm vacuole size. We showed previously that hexose
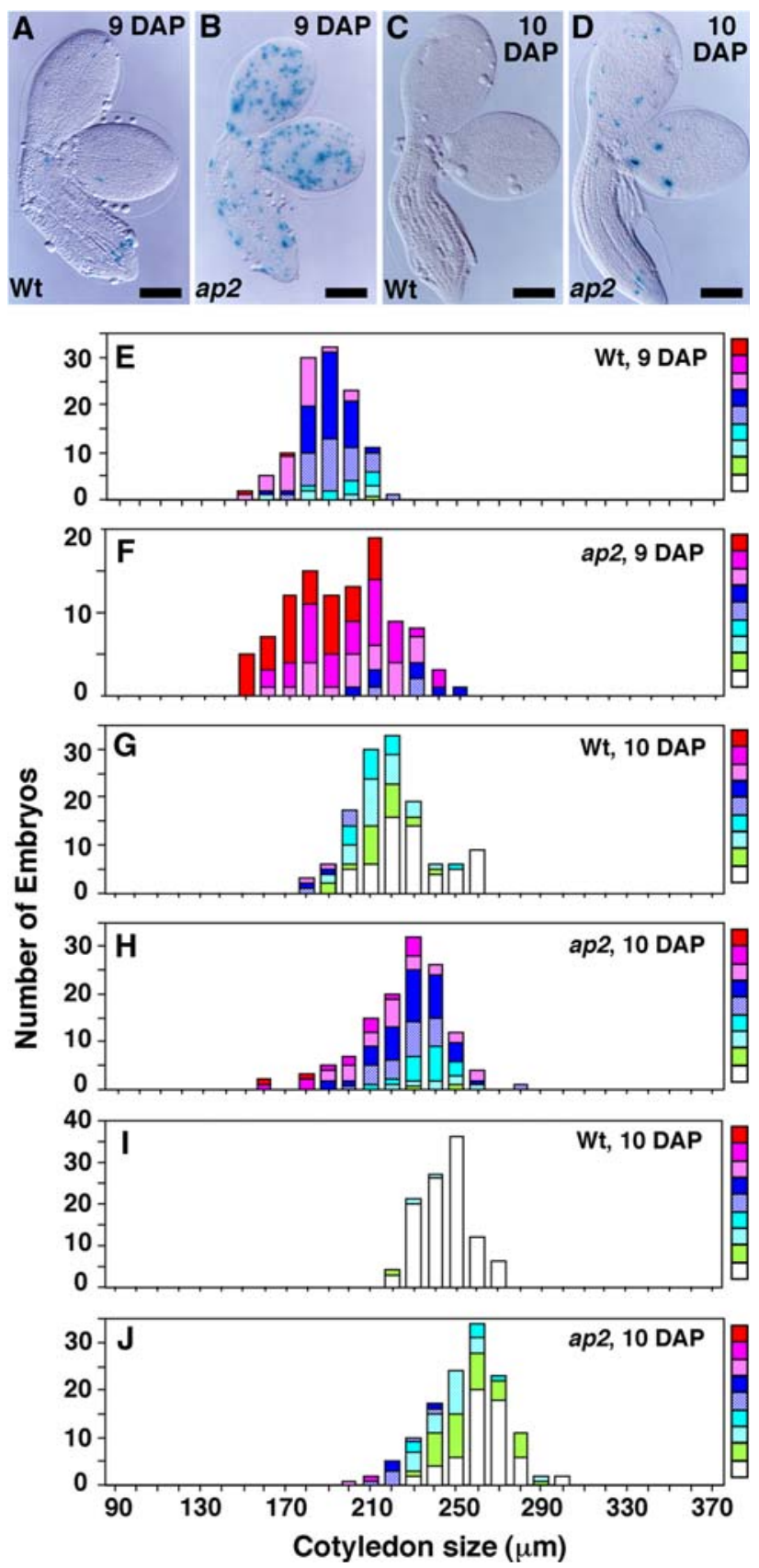

Fig. 6 ap2 mutation causes elevated and persistent $C y c B 1 ; 1: G U S$ expression during embryo development. a-d Wild-type (a, c) and ap2 (b, d) embryos with $C y c B 1 ; 1: G U S$ stained for GUS activity at the indicated DAP. e-j Embryos obtained from crosses between wildtype $(\mathbf{e}, \mathbf{g}, \mathbf{i})$ and $\operatorname{ap} 2(\mathbf{f}, \mathbf{h}, \mathbf{j})$ females and $C y c B 1 ; 1: G U S$ males were stained for GUS activity at the indicated DAP. Embryos from three to four siliques were analyzed at each time point. Similar results were obtained whether $C y c B 1 ; 1: G U S$ was contributed by the maternal or paternal parent. Data presentation is as described in Fig. 5. Bar $=100 \mu \mathrm{m}$

levels are higher in ap2 than wild-type seeds between 5 and 13 DAP during seed development (Ohto et al. 2005). We speculated that AP2 may regulate the activity of enzymes 


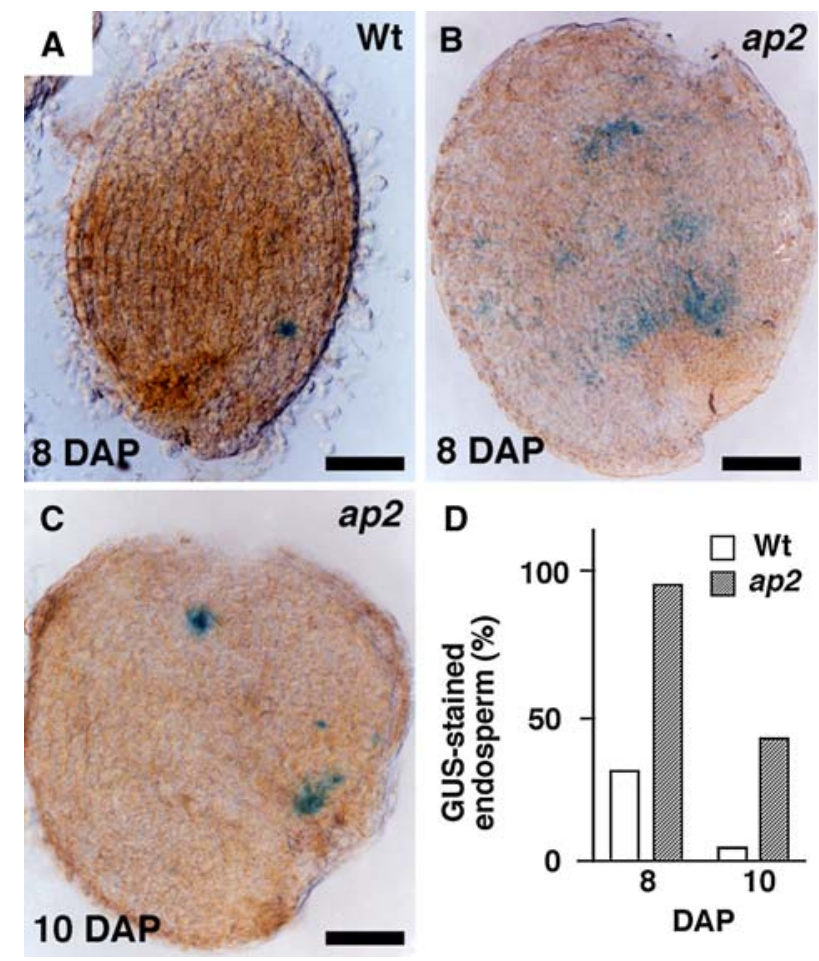

Fig. 7 Extended period of $C y c B 1 ; 1: G U S$ activity during endosperm development in ap2 mutants. a-c Endosperm and seed coat fraction of developing wild-type (a) and ap2 (b, c) seeds were stained for GUS activity at the indicated DAPs. Wild-type seeds typically showed one blue spot at $8 \mathrm{DAP}$, whereas ap2 seeds showed one to three spots at 10 DAP. d Percentage of GUS-stained endosperm/seed coats. At 8 and $10 \mathrm{DAP}, n=120$ and 117 , respectively, for wild-type endosperm/seed coat and $n=124$ and 135, respectively, for ap2 endosperm/seed coats. Seeds were derived from a cross between wild-type or ap2 mutant females and wild-type males: all plants were homozygous for $C y c B 1 ; 1: G U S$. Bar $=100 \mu \mathrm{m}$

involved in converting sucrose to hexoses in the seed coat, such as cell wall-bound invertases, thus controlling the composition of maternally supplied sugars transported to the embryo and endosperm, although another report suggests sugar composition is determined within the endosperm vacuole in B. napus (Morley-Smith et al. 2008). It is possible that the increased size of the ap 2 endosperm vacuole relative to wild type observed at 6 and 7 DAP (Fig. 4) may result in part from higher hexose accumulation in ap 2 versus wild type endosperm vacuoles. Consistent with this possibility, a recent report suggests that hexose derived from maternally supplied sucrose accumulates primarily within the endosperm vacuole early in Brassica napus seed development (Morley-Smith et al. 2008). A second potential explanation derives from our observation that integument cells are longer in ap2 seed coats compared with wild type. Thus, AP2 may directly suppress integument cell elongation to limit expansion of the postfertilization embryo sac, potentially restricting endosperm size.
AP2 affects embryo mass

AP2 suppresses embryo size, affecting both embryo cell number and size (Jofuku et al. 2005; Ohto et al. 2005). We hypothesize that the suppression of endosperm and/or seed coat growth by AP2 limits the volume of the postfertilization embryo sac, thus creating a physical constraint limiting embryo growth. Consistent with this idea, both ap2 and wild-type embryos completely fill their respective postfertilization embryo sacs at maturity even though the embryos differ in size (Fig. 1). An alternate possibility, not exclusive of the former, is that because the endosperm supplies nutrients for the developing embryo (Lopes and Larkins 1993; Hirner et al. 1998; Baud et al. 2005), a smaller endosperm limits resource availability, restricting embryo growth.

Our results suggest that the period of embryo cell proliferation is extended in ap 2 mutants relative to wild type (Fig. 6), likely accounting for the larger number of cells in the ap 2 embryo. This longer period of embryo cell proliferation could result from the effect of the ap 2 mutation on sugar composition in seeds. Studies with favabean embryos determined that a high hexose to sucrose ratio promotes cell proliferation during the morphogenesis phase (Weber et al. 1996). ap2 mutants maintain a high hexose to sucrose ratio later in seed development than wild-type seeds, and the extended period of cell proliferation in ap2 embryos correlates with the time period in which the hexose to sucrose ratio remains high (Ohto et al. 2005). Although a recent report inferred that $B$. napus embryos were not directly exposed to the high hexose to sucrose ratio characteristic of whole seeds (Morley-Smith et al. 2008), we showed wild-type embryos cultured under high hexose to sucrose conditions continued to proliferate, whereas those cultured in high sucrose did not (Fig. 8).

The larger cells of mature ap 2 embryos may be a consequence of the extended period of seed development in ap2 mutants. Although the maturation phase, as judged by the activities of storage protein and oleosin promoters, begins approximately 1 day later in ap 2 embryos, the total length of seed development is extended from 17.5 days in wild type to 20 days in ap2 seeds. Consistent with this observation, wild-type and ap 2 embryos are approximately the same size at 8-9 DAP (Figs. 1, 5, 6), whereas mature ap2 embryos are approximately 1.8 -fold larger than wild type (Ohto et al. 2005). The increase in ap2 embryo size relative to wild type occurred primarily during the maturation phase after 8-9 DAP, consistent with the finding ap2 seeds possess more storage protein and lipid than wild type (Jofuku et al. 2005; Ohto et al. 2005). Thus, ap2 embryos have a longer period to accumulate storage reserves and undergo cell expansion, potentially accounting for the increase in embryo cell size. 
Fig. 8 Effect of sugar composition on $C y c B 1 ; 1:: G U S$ activity in developing embryos. Excised wild-type embryos with $C y c B 1 ; 1:: G U S$ at 8 DAP were cultured in medium with a high ratio of hexose to sucrose (a) or high sucrose concentration (b). Approximately 48, 37, and 15\%, respectively, of embryos cultured in medium with a high hexose to sucrose ratio exhibited high (similar to (a)), medium, and low (similar to (b)) staining, whereas embryos cultured in high sucrose medium displayed either low $(87 \%)$ or medium $(13 \%)$ intensity staining. Bar $=100 \mu \mathrm{m}$
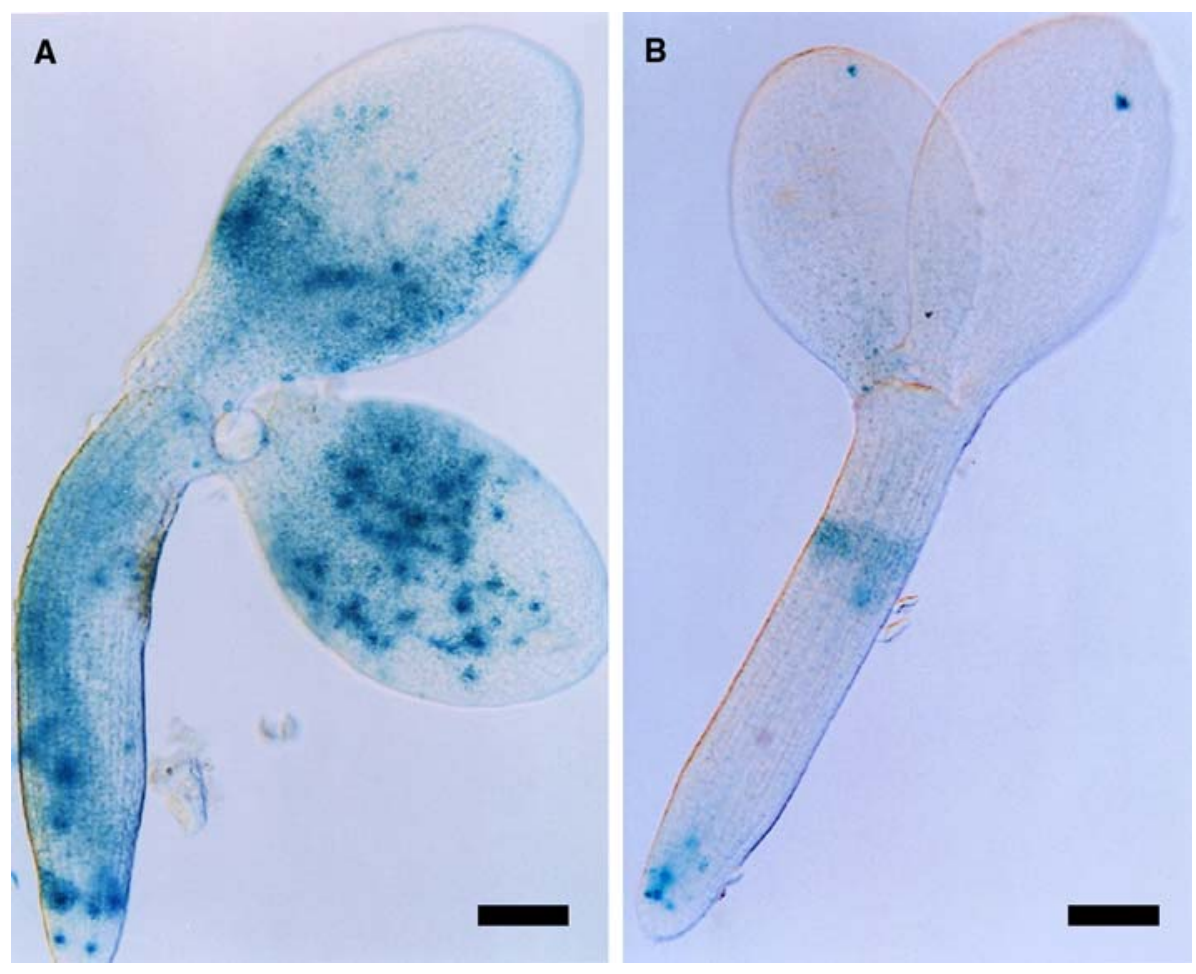

Table 3 Endosperm nuclei do not overproliferate in ap2 seeds

\begin{tabular}{llccc}
\hline Genotype & DAP & Endosperm nuclei no. ${ }^{\mathrm{a}}$ & Embryo size $^{\mathrm{a}}$ & Embryo stage \\
\hline WT & 3 & $133 \pm 37^{\mathrm{c}}$ & $29.5 \pm 3.6^{\mathrm{d}, \mathrm{e}}$ & Globular \\
ap 2-7 & 3 & $84 \pm 27^{\mathrm{c}}$ & $24.2 \pm 2.5^{\mathrm{d}, \mathrm{e}}$ & Octant/globular \\
WT & 6 & $394 \pm 11$ & $212 \pm 20^{\mathrm{f}}$ & Linear cotyledon \\
ap2-7 & 6 & $399 \pm 11$ & $215 \pm 12^{\mathrm{f}}$ & Linear cotyledon \\
\hline
\end{tabular}

${ }^{\mathrm{a}}$ Mean \pm standard deviation

${ }^{b}$ Number of seeds

${ }^{c}$ Differs at the $<0.001$ significance level

${ }^{d}$ Differs at the $<0.001$ significance level

e Width of embryo proper

${ }^{\mathrm{f}}$ Length of embryo was measured

g ap2 seeds with the most developmentally advanced embryos and the highest number of endosperm nuclei were used

\section{Conclusions}

We have shown that AP2 acts to control seed size through its effects on embryo, endosperm and seed coat development. AP2 represses endosperm growth early in seed development, potentially by limiting endosperm vacuole growth, controlling the timing of endosperm cellularization, and/or by restricting seed coat integument cell elongation. We propose that endosperm growth and/or integument cell elongation determine the size of the postfertilization embryo sac, which places a physical constraint on embryo growth. AP2 appears to influence embryo growth both by controlling the duration of the cell proliferation phase and the length of the maturation phase late in seed development.

We and others showed previously that AP2 acts maternally to control seed size, although another report suggested that AP2 activity in the endosperm has a minor effect on seed size (Jofuku et al. 2005; Ohto et al. 2005). Our genetic studies confirmed that AP2 acts independently of parent-oforigin effects on DNA methylation in controlling seed size (Fig. 9). Interactions between the maternal seed coat and 


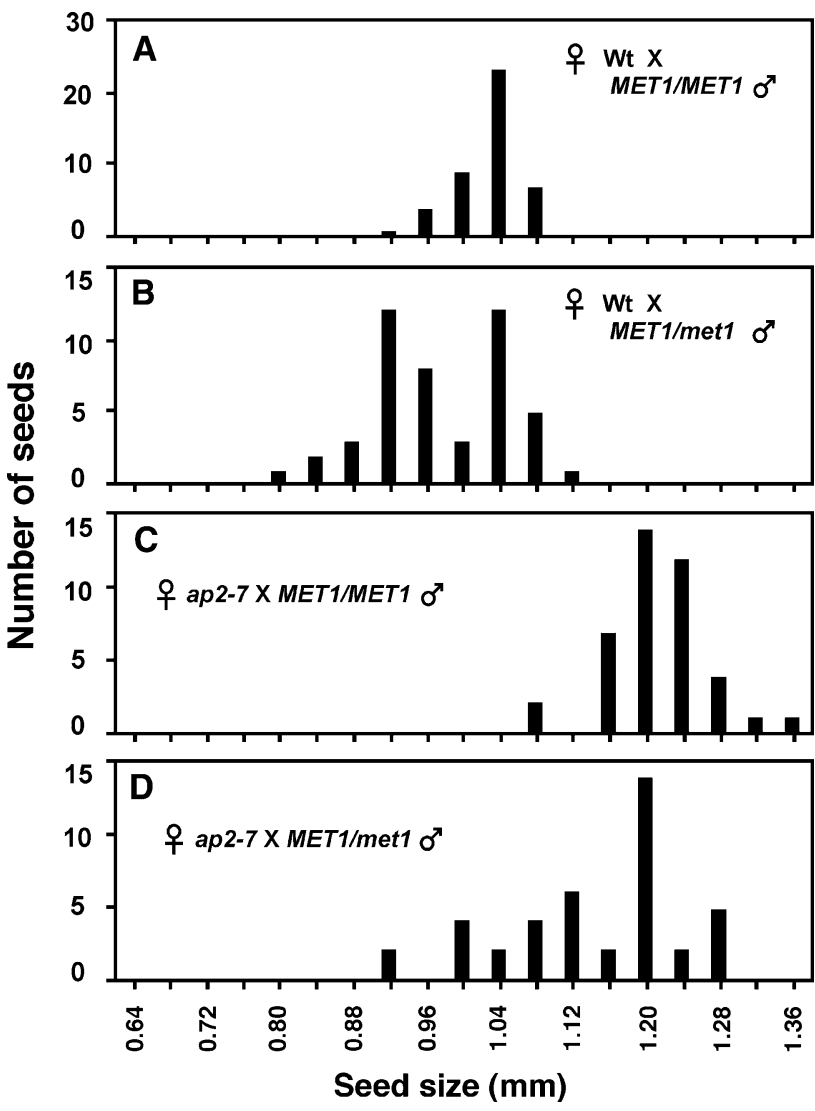

Fig. 9 Effects of hypomethylation of pollen DNA on seed size in ap2 mutants and wild type. Histograms show the distributions of seed sizes at 13 DAP obtained from crosses between the following genotypes. a Wild-type female, homozygous wild-type male. b Wild-type female, heterozygous met 1 mutant male. c ap 2 mutant female, wild-type male. d ap 2 mutant female, heterozygous met 1 mutant male

zygotic embryo and endosperm have been shown to affect seed size (Garcia et al. 2005; Ungru et al. 2008). However, it is unclear how AP2 act maternally to affect embryo and endosperm development. One possibility is that the effects of AP2 on sugar composition, potentially within the seed coat, could be a common factor underlying the control of endosperm and embryo growth. This possibility is consistent with other reports showing that hexose to sucrose ratios early in seed development appear to be controlled by the maternal seed coat in legumes (reviewed by Weber et al. 2005). Alternatively, AP2 could affect seed size by controlling integument cell elongation in the seed coat. Additional experiments are needed to understand how AP2 affects seed development and seed size at a mechanistic level.

Acknowledgments We thank Peter Doerner (University of Edinburgh) for $C y c B 1 ; 1:: G U S$, Satoshi Naito (Hokkaido University) for $\beta C G$ :GUS, Wenyan Xiao (UC Berkeley) for met1-6, and the Arabidopsis Biological Resource Center for ap2-7 seeds. This work was supported in part by grants from the Department of Energy, National Science Foundation, and Ceres Inc. to J.J.H.
Open Access This article is distributed under the terms of the Creative Commons Attribution Noncommercial License which permits any noncommercial use, distribution, and reproduction in any medium, provided the original author(s) and source are credited.

\section{References}

Adams S, Vinkenoog R, Spielman M, Dickinson HG, Scott RJ (2000) Parent-of-origin effects on seed development in Arabidopsis thaliana require DNA methylation. Development 127:2493-2502

Baud S, Wuilleme S, Lemoine R, Kronenberger J, Caboche M, Lepiniec L, Rochat C (2005) The AtSUC5 sucrose transporter specifically expressed in the endosperm is involved in early seed development in Arabidopsis. Plant J 43:824-836

Bewley JD, Black M (1994) Seeds: physiology of development and germination. Plenum Publishing Corporation, New York

Boisnard-Lorig C, Colon-Carmona A, Bauch M, Hodge S, Doerner P, Bancharel E, Dumas C, Haseloff J, Berger F (2001) Dynamic analyses of the expression of the HISTONE:YFP fusion protein in Arabidopsis show that syncytial endosperm is divided in mitotic domains. Plant Cell 13:495-509

APETALA1 Bowman JL, Smyth DR, Meyerowitz EM (1991) Genetic interactions among floral homeotic genes of Arabidopsis. Development 112:1-20

Bowman JL, Alvarez J, Weigel D, Meyerowitz EM, Smyth DR (1993) Control of flower development in Arabidopsis thaliana by and interacting genes. Development 119:721-743

Brown RC, Lemmon BE, Nguyen H, Olsen OA (1999) Development of endosperm in Arabidopsis thaliana. Sex Plant Reprod 12:32

Colon-Carmona A, You R, Haimovitch-Gal T, Doerner P (1999) Spatio-temporal analysis of mitotic activity with a labile cyclinGUS fusion protein. Plant J 20:503-508

Dietrich RA, Maslyar DJ, Heupel RC, Harada JJ (1989) Spatial patterns of gene expression in Brassica napus seedlings: identification of a cortex-specific gene and localization of messenger RNA encoding isocitrate lyase and a polypeptide homologous to proteinases. Plant Cell 1:73-80

Floyd SK, Lerner VT, Friedman WE (1999) A developmental and evolutionary analysis of embryology in Platanus (platanaceae), a basal eudicot. Am J Bot 86:1523-1537

Garcia D, Saingery V, Chambrier P, Mayer U, Jurgens G, Berger F (2003) Arabidopsis haiku mutants reveal new controls of seed size by endosperm. Plant Physiol 131:1661-1670

Garcia D, Fitz Gerald JN, Berger F (2005) Maternal control of integument cell elongation and zygotic control of endosperm growth are coordinated to determine seed size in Arabidopsis. Plant Cell 17:52-60

Haig D, Westoby M (1991) Genomic imprinting in the endosperm: its effect on seed development in crosses between species, and between different ploidies of the same species, and its implications for the evolution of apomixis. Philos Trans R Soc Lond B Biol Sci 333:1-13

Harada JJ (1997) Seed maturation and control of germination. In: Larkins BA, Vasi IK (eds) Advances in cellular and molecular biology of plants. Cellular and molecular biology of seed development, vol 4. Kluwer Academic Publishers, Dordrecht, pp 545-592

Hirai MY, Fujiwara T, Goto K, Komeda Y, Chino M, Naito S (1994) Differential regulation of soybean seed storage protein gene promoter-gus fusions by exogenously applied methionine in transgenic Arabidopsis thaliana. Plant Cell Physiol 35:927-934

Hirner B, Fischer WN, Rentsch D, Kwart M, Frommer WB (1998) Developmental control of $\mathrm{H}+$ /amino acid permease gene 
expression during seed development of Arabidopsis. Plant J $14: 535-544$

Irish VF, Sussex IM (1990) Function of the apetala-1 gene during Arabidopsis floral development. Plant Cell 2:741-753

Jofuku KD, Boer B, Montagu MV, Okamuro JK (1994) Control of Arabidopsis flower and seed development by the homeotic gene APETALA2. Plant Cell 6:1211-1225

Jofuku KD, Omidyar PK, Gee Z, Okamuro JK (2005) Control of seed mass and seed yield by the floral homeotic gene APETALA2. Proc Natl Acad Sci USA 102:3117-3122

Kang IH, Steffen JG, Portereiko MF, Lloyd A, Drews GN (2008) The AGL62 MADS domain protein regulates cellularization during endosperm development in Arabidopsis. Plant Cell 20:635647

Kunst L, Klenz JE, Martinez-Zapater J, Haughn GW (1989) AP2 gene determines the identity of perianth organs in flowers of Arabidopsis thaliana. Plant Cell 1:1195-1208

Kwong RW (2003) Control of Arabidopsis thaliana embryogenesis and the developmental regulation of embryo identity at the transcriptional level. Dissertation, University of California, Davis

Lopes MA, Larkins BA (1993) Endosperm origin, development, and function. Plant Cell 5:1383-1399

Luo M, Bilodeau P, Dennis ES, Peacock WJ, Chaudhury A (2000) Expression and parent-of-origin effects for FIS2, MEA, and FIE in the endosperm and embryo of developing Arabidopsis seeds. Proc Natl Acad Sci USA 97:10637-10642

Millerd A, Spencer D, Dudman WF, Stiller M (1995) Growth of immature pea cotyledons in culture. Aust J Plant Physiol 2:5159

Morley-Smith ER, Pike MJ, Findlay K, Koeckenberger W, Hill LM, Smith AM, Rawsthorne S (2008) The transport of sugars to developing embryos is not via the bulk endosperm in oilseed rape seeds. Plant Physiol 147:2121-2130

Ohto M, Fischer RL, Goldberg RB, Nakamura K, Harada JJ (2005) Control of seed mass by APETALA2. Proc Natl Acad Sci USA 102:3123-3128

Ohto M, Stone SL, Harada JJ (2008) Genetic control of seed development and seed mass. In: Bradford KJ, Nonogaki H (eds) Seed development, dormancy and germination. Blackwell, Oxford, pp 1-24

Okamuro JK, den Boer BGW, Jofuku KD (1993) Regulation of Arabidopsis flower development. Plant Cell 5:1183-1193
Olsen OA (2004) Nuclear endosperm development in cereals and Arabidopsis thaliana. Plant Cell 16(suppl.):S214-S227

Raz V, Bergervoet JHW, Koornneef M (2001) Sequential steps for developmental arrest in Arabidopsis seeds. Development 128:243-252

Schruff MC, Spielman M, Tiwari S, Adams S, Fenby N, Scott RJ (2006) The AUXIN RESPONSE FACTOR 2 gene of Arabidopsis links auxin signalling, cell division, and the size of seeds and other organs. Development 133:251-261

Schultz EA, Haughn GW (1993) Genetic analysis of the floral initiation process (FLIP) in Arabidopsis. Development 119:745765

Scott RJ, Spielman M, Bailey J, Dickinson HG (1998) Parent-oforigin effects on seed development in Arabidopsis thaliana. Development 125:3329-3341

Ungru A, Nowack MK, Reymond M, Shirzadi R, Kumar M, Biewers S, Grini PE, Schnittger A (2008) Natural variation in the degree of autonomous endosperm formation reveals independence and constraints of embryo growth during seed development in Arabidopsis thaliana. Genetics 179:829-841

Vicente-Carbajosa J, Carbonaro P (2005) Seed maturation: developing an intrusive phase to accomplish a quiescent state. Int J Plant Sci 49:645-651

Vinkenoog R, Spielman M, Adams S, Fischer RL, Dickinson HG, Scott RJ (2000) Hypomethylation promotes autonomous endosperm development and rescues postfertilization lethality in fie mutants. Plant Cell 12:2271-2282

Weber H, Borisjuk L, Wobus U (1996) Controlling seed development and seed size in Vicia faba: a role for seed coat-associated invertases and carbohydrate state. Plant J 10:823-834

Weber H, Borisjuk L, Wobus U (2005) Molecular physiology of legume seed development. Annu Rev Plant Biol 56:253-279

West MAL, Matsudaira Yee KL, Danao J, Zimmerman JL, Fischer RL, Goldberg RB, Harada JJ (1994) LEAFY COTYLEDON1 is an essential regulator of late embryogenesis and cotyledon identity in Arabidopsis. Plant Cell 6:1731-1745

Xiao W, Gehring M, Choi Y, Margossian L, Pu H, Harada JJ, Goldberg RB, Pennell RI, Fischer RL (2003) Imprinting of the MEA polycomb gene is controlled by antagonism between MET1 methyltransferase and DME glycosylase. Dev Cell 5:891

Xiao W, Brown RC, Lemmon BE, Harada JJ, Goldberg RB, Fischer RL (2006) Regulation of seed size by hypomethylation of maternal and paternal genomes. Plant Physiol 142:1160-1168 